\title{
Towards cyclic fabrication systems for modular robotics and rapid manufacturing
}

\author{
Matthew S. Moses*, Hiroshi Yamaguchi ${ }^{\dagger}$, Gregory S. Chirikjian ${ }^{\ddagger}$ \\ Department of Mechanical Engineering \\ Whiting School of Engineering, \\ Johns Hopkins University, \\ Baltimore, Maryland 21218-2682 \\ Email: matt.moses* ${ }^{*}$ hyamaguchi ${ }^{\dagger}$, gregc $^{\ddagger} @$ jhu.edu
}

\begin{abstract}
A cyclic fabrication system (CFS) is a network of materials, tools, and manufacturing processes that can produce all or most of its constituent components. This paper proposes an architecture for a robotic CFS based on modular components. The proposed system is intended to self-replicate via producing necessary components for replica devices. Some design challenges unique to self-replicating machines are discussed. Results from several proof-of-principle experiments are presented, including a manipulator designed to handle and assemble modules of the same type it is constructed from, a DC brush motor fabricated largely from raw materials, and basic manufacturing tools made with a simple CFS.
\end{abstract}

\section{INTRODUCTION}

In the most general terms, we are interested in "making machines that make others of their kind" [1]. This topic, first formally introduced by von Neumann and Burks [2] has seen sporadic interest over ensuing decades with a recent increase in research activity. Applications of self-replicating machines have been proposed ranging from space exploration [3] [4], construction of solar power arrays [5], desktop rapid manufacturing [6] [7] [8], and nanotechnology. Reviews of much of this research are presented in [9] [10].

For the purpose of discussion it is helpful to classify the process of "making" into "assembly" and "fabrication". Assembly involves the formation of kinematic constraints between prefabricated components (e.g. nuts and bolts, keyways, snapconnectors, magnetic latches), while fabrication involves forming useful components from raw materials via changes in molecular structure (e.g. casting, welding, soldering, gluing, cutting, bending). Most recent research falls nicely into one category or the other. In the assembly category, researchers have demonstrated general processes of shape change through reconfiguration [11] [12] [13], stochastic assembly of random components [14] [15], prototype assembly systems for MEMS [16], and self-replication through centrally directed assembly of modular robots [17] [18] [19]. Within fabrication, two groups are developing desktop printers with the potential to replicate their constituent components [6] [7], and other groups are using rapid manufacturing machines to produce fully formed robotic devices [20] [21]. Research on self-assembly of meso-scale mechanical components falls at the boundary between fabrication and assembly [22]. There is ongoing activity in the simulation of self-replicating machines, using

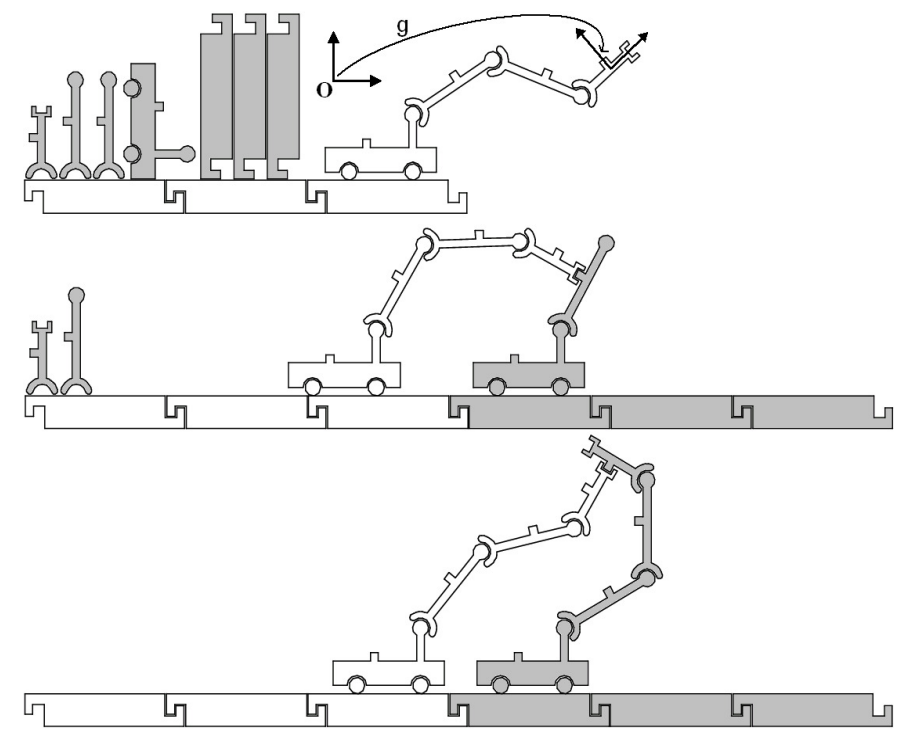

Fig. 1. Schematic representation of assembly during self-replication. Prefabricated components are stacked in a known arrangement. The task of the mobile manipulator is to extend the structural lattice and assemble a new manipulator from the stacked components.

both cellular automata [23] and physics based simulations [24] [25]. There is also work on theoretical aspects of selfreplicating machines [26] [27] [28].

This paper proposes an architecture for a selfreconfigurable, self-replicating manufacturing system based on a set of "universal" electro-mechanical components. Section II discusses some of the challenges that generally arise in self-replicating machine design. Section III illustrates one particular approach to the problem and presents a conceptual vision for what a completed solution would look like. Section IV presents results from several proof-ofprinciple experiments performed to verify elements of the overall system design.

\section{Problem Formalization}

While the topics of robot assembly planning and automated manufacturing are well-studied, there are certain complications introduced when a robot must build a replica of itself. The common theme across these complications is that the robot 


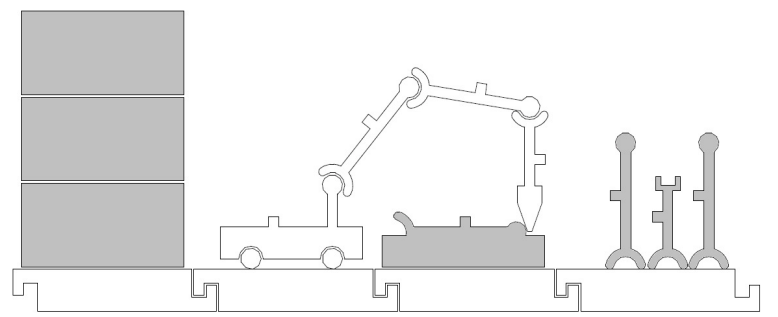

Fig. 2. Schematic representation of fabrication. A robot manipulator equipped with material deposition/removal nozzles fabricates components from a source of raw materials. Fabricated components are then made available to the assembly system.

and the thing it builds cannot be "decoupled". In industrial practice, a product may be designed to suit the capabilities of a certain robot, or a robot may be modified to perform a specialized task. In the case of self-replication, the robot and the product cannot be independently modified - a change to one necessitates a change to the other. This section discusses some of these complications as they arise in assembly and fabrication processes in machine self-replication.

\section{A. Assembly}

A schematic of the assembly process is shown in Figure 1. A mobile manipulator has access to a set of prefabricated components arranged in known configurations. The task of the robot is to obtain components and assemble them into a duplicate robot. The robot begins by constructing an extension to the platform, and then assembles a duplicate manipulator in the new space.

1) Workspace: The assembly robot must be able to build a device as large as itself. For each component in the robot, there must be a collision-free path between the part storage location and the part's destination on a growing replica. Numerous approaches have been reported for dealing with the workspace problem. For example, [19] and [29] actuate the partiallyconstructed replica during assembly so that the original machine may access necessary areas. In contrast [17] and [18] use mobile manipulators operating in an environment common to original and replica. The environment guides the original robot around the growing replica, effectively increasing the workspace.

2) Assembly Error Tolerance: A physical robot manipulator will have some error in the position of the end-effector, due to imperfections in measurement and manufacturing. For a single link, this positioning error can be represented as a probability density function $\rho(g)$ on the group of rigid-body transformations $g \in S E(3)$. A pdf for the entire manipulator, representing positional uncertainty of the end-effector, can be generated by "concatenating" the error distributions of individual links, using convolutions of the form [30]

$$
\rho_{12}(g)=\left(\rho_{1} * \rho_{2}\right)(g)=\int_{G} \rho_{1}(h) \rho_{2}\left(h^{-1} \circ g\right) d h .
$$

The allowable assembly tolerance between two mechanical parts can be represented as a function $\alpha(g)$ on $g \in S E(3)$.

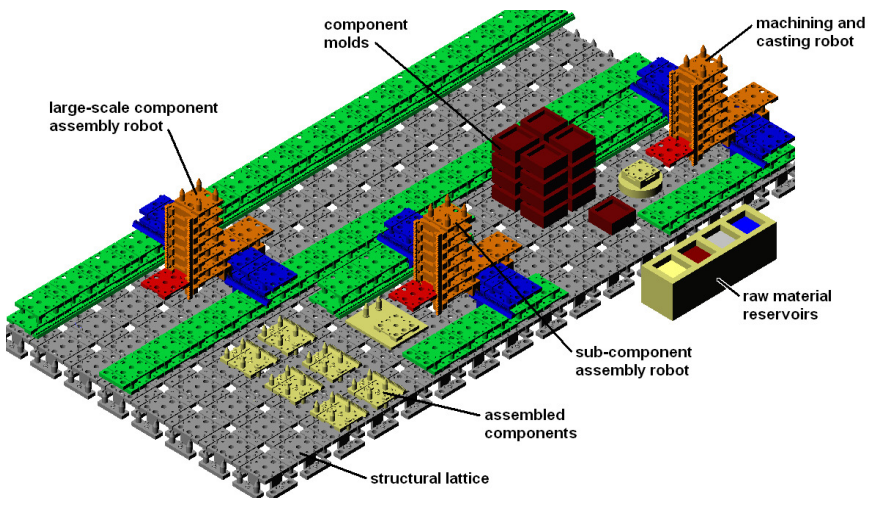

Fig. 3. Concept of a large-scale automated cyclic fabrication system. Mobile 3-axis manipulators operate on a structural grid, fabricating components from raw materials and then adding them to the grid and building additional machines.

This function varies between 0 and 1 and represents the likelihood of a successful connection when the two components are placed with relative configuration $g$. The overall likelihood that a given manipulator can successfully assemble two components is given by the integral

$$
\gamma=\int_{G} \alpha(g) \rho(g) d g .
$$

An important design goal is to maximize $\gamma$.

Intuitively, it is good for the manipulator to have a tight distribution, the optimal being the delta function $\rho(g)=$ $\delta(g)$. It is also desirable for the mechanical parts to tolerate large relative displacements. One measure of the uncertainty tolerance in the relative part configurations is parts entropy [31]

$$
S=-\int_{G} \bar{\alpha}(g) \log \bar{\alpha}(g) d g,
$$

where $\bar{\alpha}(g)$ indicates $\alpha(g)$ normalized to a pdf. A large $S$ corresponds to a greater tolerance of uncertainty and is hence desirable. Note that $\rho(g)$ is conditioned on design parameters of the parts themselves, which can be represented as a vector a, and on the arrangement of parts in the manipulator, which can be represented as a vector of parameters $\mathbf{b}$. The assembly tolerance function $\alpha(g)$ is itself a function of the part parameters $\mathbf{a}$. The dependence of $\gamma$ on $\mathbf{a}$ and $\mathbf{b}$ can be written as

$$
\gamma(\mathbf{a}, \mathbf{b})=\int_{G} \alpha(g, \mathbf{a}) \rho(g \mid \mathbf{a}, \mathbf{b}) d g .
$$

This illustrates the "coupling" between the robot and what it builds, as both $\rho$ and $\alpha$ depend on the same parameter vector a. Challenges arise because changing a to induce a desirable change in $\alpha$ can induce undesirable changes in $\rho$, resulting in minimal or detrimental impact to the overall function $\gamma$, which is what should actually be maximized.

The set of components described in Section IV-A are designed largely by engineering intuition. However, this formalism provides a way to approach the design process in a more systematic way. A topic of current study is the quantification 
of $\rho(g \mid \mathbf{a}, \mathbf{b}), \alpha(g, \mathbf{a})$, and $\gamma(\mathbf{a}, \mathbf{b})$, using data collected over many assembly trials with various part designs.

3) Connector Mechanism Design: Connector design is a challenging problem in all types of reconfigurable modular robot systems. A standard connection mechanism must be devised that provides mechanical strength and electrical interconnection between modules. Additionally, the connector usually also functions as a "handle" so that modules may be grasped and manipulated using a standard end-effector. It is important to keep the connector as simple as possible, because the connector must be built by other parts of a selfreplicating system. A more complex connector may solve certain assembly problems, but it introduces other problems during the fabrication steps.

\section{B. Fabrication}

A schematic representation of fabrication is shown in Figure 2. The fabricating machine takes some form of refined raw materials as input (blocks, liquid resins, etc) and produces finished components as output.

1) Selection of Materials and Processes: Materials and processes must be carefully chosen so that the system can fabricate its constituent components. In industrial practice, cutting tools (for example) can be produced on special purpose machines able to form hard materials. Hardened tools are then used to form more generic products from softer materials. In order to "close the loop" in self-replication, some process must be selected in which machines made of soft materials can form hardened cutting tools.

2) Force and Temperature Limitations: Fabrication processes often require the use of large forces and temperatures, in turn requiring heavy, high-precision machinery. In general, it is desirable to select manufacturing processes that use forces and temperatures that are as low as possible, as this simplifies the associated machinery, which of course must be built by some part of the self-replicating system.

3) Component Sub-assembly: Ideally the fabricating robot could simply print entire functional components to be used in assembly. However in many cases it is necessary for several fabricated components to be sub-assembled into a component suitable for use by the assembly system.

4) Resolution Limitations: The problem of resolution is somewhat analogous to the assembly tolerance problem (Section II-A.2). The components produced by the fabricating system must be functional even though they are produced to rather crude tolerances. Because the system self-replicates, the range of acceptable part tolerances must be the same for both the fabricating machine and the products it fabricates.

5) Generational Error Correction: Generational error correction really applies to the entire system. We place it in the fabrication category because it is assumed that given suitably fabricated components, the assembly error tolerance built-in to the modules allows the assembly system to produce functional robots at some guaranteed success rate. Only a few studies have looked at this topic [27]. It is clear that some type of error correction must be built in to the fabrication system to avoid

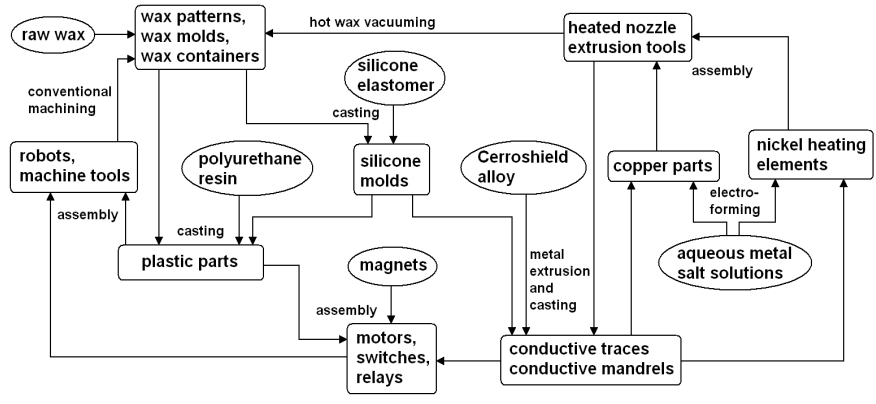

Fig. 4. Materials, tools, and manufacturing processes comprise a cyclic fabrication system. Raw materials are shown in ellipses, products are shown in boxes, and processes are indicated by labeled arrows.

accumulation of errors over successive replications. This could be performed by some intergenerational calibration methods, or by calibration to some external standard.

\section{CONCEPT OVERVIEW}

The model system we use for inspiration is something most engineers are familiar with: LEGO $^{\mathrm{TM}}$. Modern LEGOs can be made into many kinds of robots and automated devices. They are mentioned by name in some of the early papers on modular robotics [32]. Several LEGO systems have been demonstrated that assemble simple devices also made from LEGOs [33] [34]. It is a much harder task to build a LEGO machine that can fabricate LEGO blocks. To state the goal of this work in simple terms, we seek to build a set of "LEGO-like" blocks, such that a machine made from them can 1) assemble complex devices from similar blocks, and 2) produce new blocks from a stock of raw materials. Ultimately, a fabricating machine built from this set of blocks should be able to fabricate all the essential components in the set, including sensors, actuators, and control devices.

\section{A. Lattice Architecture}

Figure 3 shows a concept of how the fabrication system might be implemented. Multiple mobile manipulator robots are installed on a structural grid. Each robot is based on a standardized 3-axis Cartesian manipulator with a general purpose end effector. An external system provides power and control. Tasks are classified into fabrication, mid-level component assembly, and large-scale structural assembly. The fabrication sub-system obtains raw materials from reservoirs and produces basic parts. These parts are then assembled into larger components by the mid-level assembly robot. The largescale assembly robot assembles components into new devices (e.g. new robots and new areas of structural lattice).

The system as a whole can self-replicate through a gradual process of growth. An initial system with a given lattice structure and a certain number of robots replicates by increasing the lattice size and doubling the number of robots. Manufacturing output is not limited to strict replication; the system can grow and reconfigure in a flexible manner. For example, the large-scale assembly robots can also reconfigure 

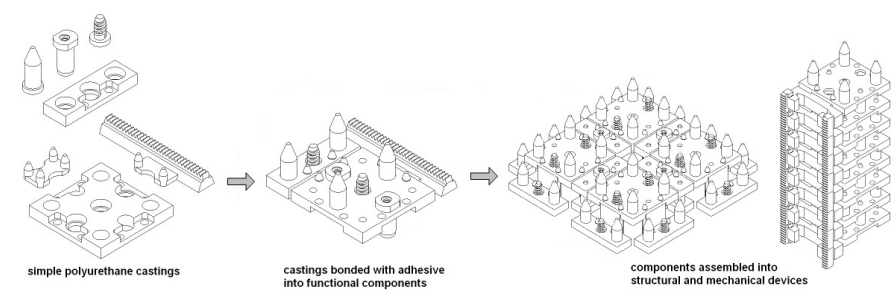

Fig. 5. Individual cast plastic parts are combined into mid-level components, which are assembled into larger structures.

their working envelope by adding and removing components in the structural grid. Depending on the application, the useful output of the system may be some product made by the robots (gears, motors, etc.), or it may be the structural grid itself. For example, some proposed applications of modular robots call for a large reconfigurable lattice [35]. A large lattice composed primarily of passive components, but reconfigured by a small number of active robots moving within the lattice, might be a feasible alternative to reconfigurable lattices in which every element is an active modular robot.

\section{B. Cyclic Fabrication Systems}

A cyclic fabrication system (CFS) is a network of materials, tools, and manufacturing processes that can produce all or most of its constituent components. It is cyclic in the way the game "rock-paper-scissors" is cyclic: tools, materials, and fabrication processes are chosen such that one process creates tools used in the next process, which is used in the next, and so on until a final process produces tools needed to perform the initial process. Complex CFSs have been proposed in the context of self-replicating machines. Processes for producing machine components from lunar soil are proposed in [3] and [4]. A cyclic process for separating common terrestrial soil into useful materials is proposed in [36]. A comparatively simple system is outlined in [37] based on UV-catalyzed polymer resin.

A proposed cyclic fabrication system is shown in Figure 4 , based on polyurethane and silicone resins, wax, lowtemperature-melting alloys, and solutions of metal salts. The primary benefit of these materials is that they are readily available and fairly safe and easy to work with in a small laboratory environment. They also serve as proxy materials for performing proof-of-principle experiments that can later be extended to materials that are harder to work with. The processes used in the system are conventional machining and assembly operations, casting, hot wax vacuuming, and electroforming.

The polyurethane resin is a two-part liquid formulation. Upon mixing, the liquid polyurethane hardens within minutes into a machineable plastic with good mechanical properties. Solid polyurethane can form a wide range of components including gears, bushings, springs, and structural members. The wax has a low melting point, is dimensionally stable and easily machined. The key features of these materials are that the polyurethane in liquid form can easily be cast

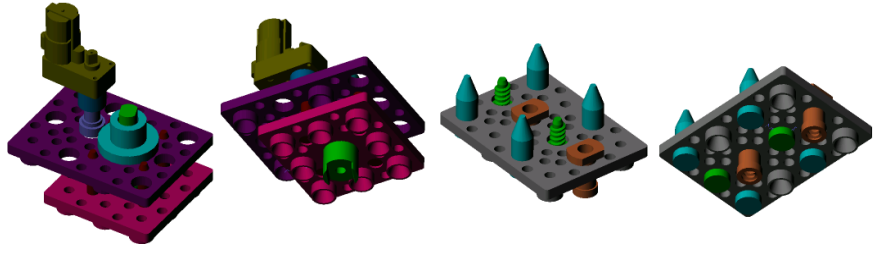

Fig. 6. The base component has four tapered compression pins, two threaded tension pins, and two captured-nut fasteners. A common end-effector is used to grasp components, and tighten/untighten them from an assembly.

in wax molds, while the wax can easily be machined by solid polyurethane tools. Master patterns made from wax can be used to form polyurethane and silicone molds, which in turn can be used to cast metal parts from low-temperature melting alloys. The resultant metal parts may be used directly in machines or as conductive mandrels for electroforming. Copper, nickel, and many other metals can be electroformed over a mandrel made of low-temperature melting alloy. The alloy can then be removed from the metal part by melting and reused. Copper can form electrical contacts, motor coils, conductive traces, and nozzles for material deposition/removal. Nickel may be used in magnetic circuits and in resistive heaters.

\section{EXPERIMENTS}

\section{A. Modular Components and Assembly Robot}

This Section presents a "universal" set of electromechanical modules. Design goals for the modules are: versatility and usefulness in assembly, ease of assembly, ease of fabrication using CFS processes, tolerance to misalignment during assembly, and strong, reversible intermodule connections. The modular components are based on an earlier design [29]. The components are made of polyurethane. Each of the castings that make up a component are designed to be produced in a single-piece wax or silicone mold with minimal undercuts. This greatly simplifies the design and fabrication of the mold, simplifies the demolding process, and eliminates difficulties that arise with removing sprues and flash. However, the exposed top surface of a part cast in a single-piece mold is not suitable for precise assembly. This problem can be solved by combining multiple parts with adhesive, so that precise dimensions between critical surfaces are maintained. With these components it is primarily achieved through the use of pins and plates. Each plate contains four compression pins, two tension pins, and two captured nuts. The compression pins are tapered and mate with tapered holes in other plates, while the tension pins mate with captured nuts.

A minimum connection between two components consists of a tension pin connected to a nut, in between two compression pins. This form of mechanical connection was chosen over other methods (magnets, snap-fittings, etc) because of its high strength and ease of reversibility. When a new component is placed on a growing assembly the tapered mating surfaces engage, locating the component. The captured nuts are then tightened onto the mating tension pins, providing a solid 


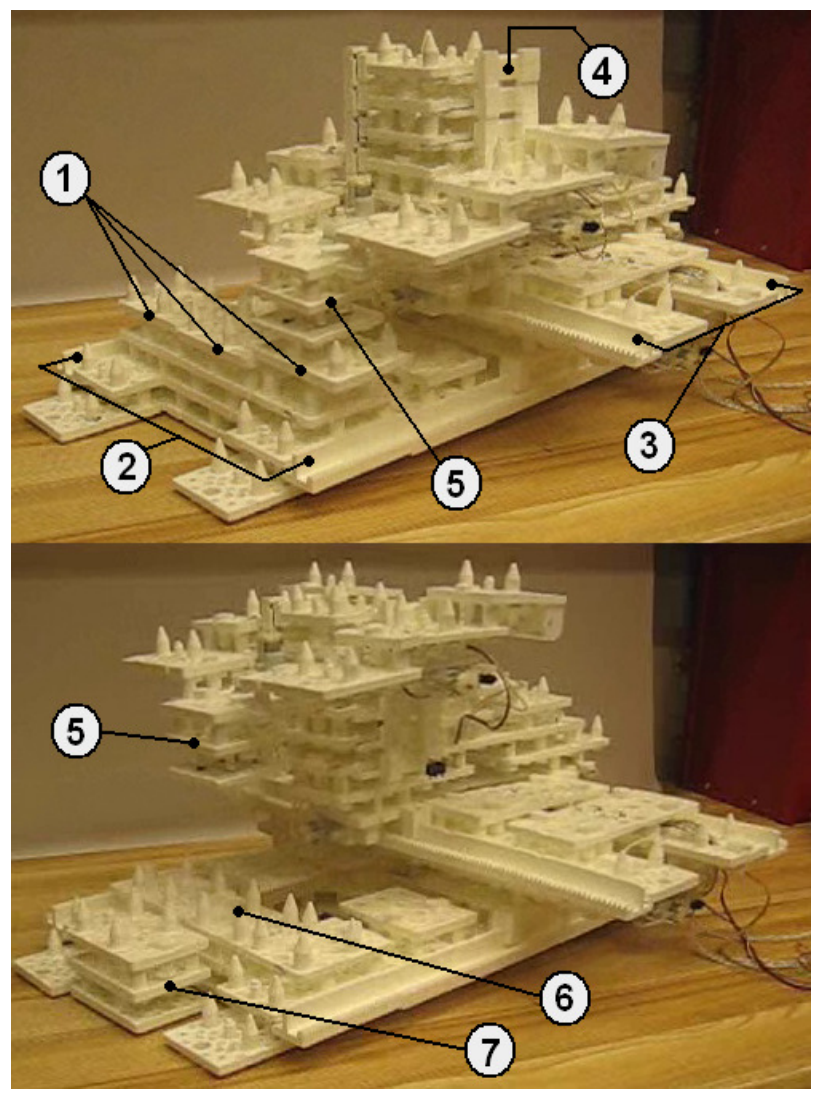

Fig. 7. A 3-axis manipulator performing a simple assembly task; (2) - X axis, (3) - Y axis, (4) - Z axis, (5) - end-effector. Three separate components (1) are initially placed on the assembly station (6). The robot assembles the three components and removes them from the assembly station (7).

mechanical connection between the new component and the assembly. Example assemblies shown in Figure 5 include a structural platform and a linear motion slide. Variations on these components can form other mechanisms, including revolute joints, gear trains, 3-axis manipulators. The threaded fasteners provide a reversible assembly mechanism, so that an existing structure may be disassembled and its parts reused in a new structure. Additional small holes in the plates are accommodations for future electrical connectors.

A single end-effector is used for grasping components and to tighten and un-tighten the threaded fasteners (see Figure 6). The end-effector consists of a spring loaded tool-piece with a slot and internal thread. For grasping a part, the internal thread of the tool mates with the threaded tension pin on a component. After a component is placed, the tool is unscrewed from the tension pin and placed on the component's capturednut fastener. The slot on the tool-piece self aligns with the fastener and tightens or loosens it in the manner of socket and nut. Apart from the spring and motor, the entire end-effector assembly is built from polyurethane components.

A 3-axis Cartesian manipulator was constructed from about 80 modular components. The manipulator is driven by seven small gearmotors (Solarbotics GM2). Two motors drive each of the axes in parallel, and the seventh is used to drive the

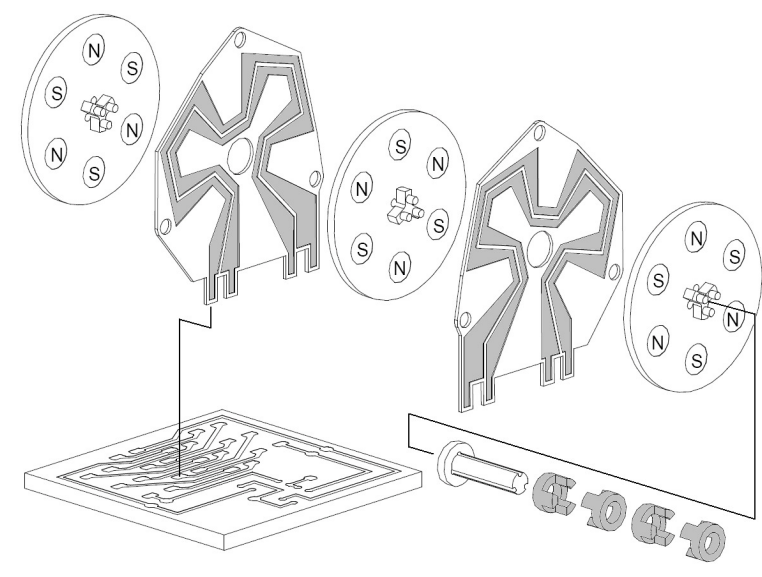

Fig. 8. Diagram of motor construction.

end-effector. Low resolution optical encoders are used on one motor on each axis for position feedback. Resolution is approximately 1 count per $\mathrm{mm}$. Power is supplied to the motors through ordinary hookup wire. The manipulator is controlled by a simple microcontroller (PIC16F690). The controller simply follows a sequence of position commands, driving each axis independently for a predetermined number of encoder counts. Due to the high tolerance for misalignment of the parts, even this crude form of control is able to automatically assemble components.

Figure 7 shows two snapshots in a test assembly sequence of the 3-axis manipulator. Three unassembled components are placed (unconnected) on an assembly station in front of the manipulator. The robot then retrieves each of the outside parts and assembles them in sequence to the center component. After the three parts are connected together, the robot lifts the assembly and removes it from the station. This is a simple example that demonstrates the feasibility of using machines made from these components to perform assembly tasks.

\section{B. Actuator Production}

This section describes a permanent magnet axial-gap Lorenz-force motor, fabricated almost entirely using techniques from the cyclic fabrication system described in Section III-B. Motors of this general design are well known, including "printed coil" motors used in many applications. The challenge here is to build such a motor using the constrained set of materials, tools, and methods that comprise the cyclic fabrication system. The Fab@home group has demonstrated printing of batteries, circuit elements, and actuators using variations on rapid manufacturing processes [38]. The Reprap group has demonstrated formation of circuit wiring by extruding metal into plastic parts [39] [40]. Inspired by their success, we designed a metal extruding mechanism with the intention of installing it on a 3-axis manipulator similar to the one in Section IV-A. The extruder consists of a heated copper nozzle and a motorized syringe pump (see Figure 9). A heated cup is used in conjunction with the nozzle device. Thermostat control of the heated nozzle and cup was performed with Reprap 


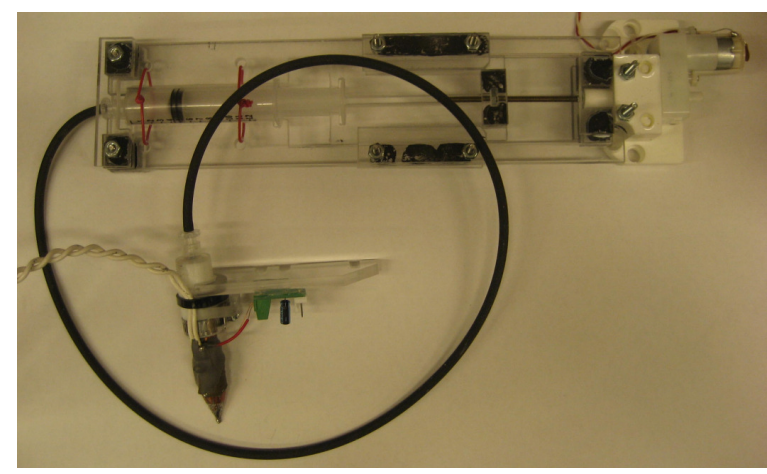

Fig. 9. Heated copper nozzle for extrusion of low-melting alloy into plastic parts.

electronics. The cup contains a small amount of molten metal, which is retrieved by the nozzle using the syringe pump. The nozzle is moved to a plastic part, where the molten metal is then pumped out into channels in the part.

1) Method of Construction: The main components of the motor are illustrated in Figures 8 and 11. The motor consists of 15 cast plastic parts - 6 coil plates, 7 magnet disks, 1 commutator shaft, 1 base plate (Smooth-On Smooth-Cast 300), 4 cast metal commutator rings (Cerroshield alloy, melting point 95 C, McMaster P/N 8921K23), 8 metal brushes (brass strip), 6 magnetic yoke pieces (mild steel strips), and $42 \mathrm{NdFeB}$ magnets (1/2" diameter by $1 / 8$ " thick, Digikey P/N 496-1002$\mathrm{ND})$. The part masters were fabricated using conventional machining and laser cutting, and the plastic and cast-metal parts were produced manually from silicone molds. To form the magnet disks, magnets were positioned in a disk mold with a plastic fixture. The fixture was then removed and liquid resin cast around the magnets to embed them in the final part. Wiring channels were cast in place as part of the coil plates and base plate. These channels were manually filled with molten Cerroshield using the heated nozzle device. During assembly, the base plate was clamped to a hot plate and coil plates with filled channels were then lowered into place onto the base plate.

2) Motor Performance: The motor can operate as a stepper motor, DC brush motor, or generator. When configured as a DC brush motor, the commutator excites the coils with a quadrature square wave. The steady-state behavior of the motor in DC operation can be described using a simple model [41]

$$
\begin{aligned}
& \tau=k_{M} i-\tau_{f}, \\
& i=\frac{V-k_{M} \omega}{R},
\end{aligned}
$$

where $\tau_{f}$ is a constant torque representing sliding friction, $V$ is constant DC voltage applied to the motor terminals, $R$ is lumped resistance of the motor windings, brushes, and commutator. The model is valid for $i>\tau_{f} / k_{M}$. For this simple motor, the characteristic we are most interested in is mechanical output power. The maximum power output in DC

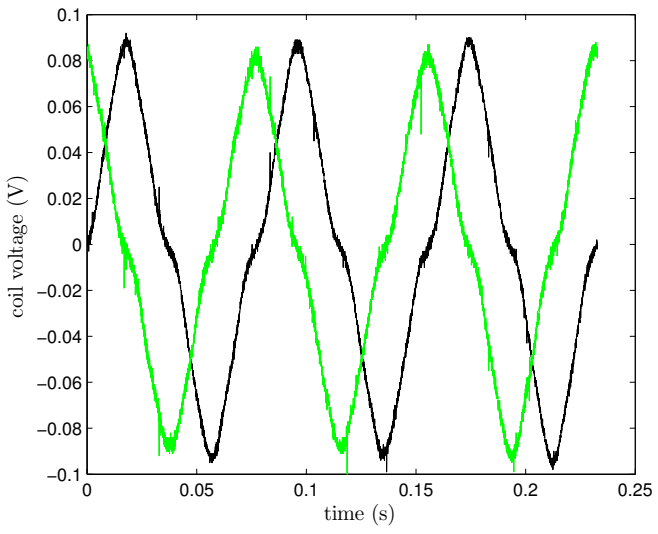

Fig. 10. Motor back-emf measured at each coil circuit. Data shown is for one revolution, $\omega=27 \mathrm{rad} / \mathrm{s}$.

motor configuration is given by

$$
P_{\text {max }}=\frac{1}{4 R}\left(V-\frac{\tau_{f} R}{k_{M}}\right)^{2} .
$$

The motor constant was measured by recording the voltage generated by the coils while the motor shaft was spun at constant speed. Figure 10 shows the voltage output of each phase in the motor over a single revolution. The measured data yields a motor constant of $k_{M}=0.00325$ (in units of $V s$ or $N m / A$ ).

The motor was tested in DC brush configuration and operated continuously for several minutes. At a terminal voltage of $6 \mathrm{~V}$ it drew about $7.5 \mathrm{~A}$ and had a no load speed of approximately $400 \mathrm{rpm}(42 \mathrm{rad} / \mathrm{s})$. The winding resistance was estimated to be $R=0.78 \Omega$, the friction torque $\tau_{f}=$ $0.024 \mathrm{Nm}$, and the maximum available power output $P_{\max }=$ $6 m W$. When compared with the commercial motor used in the assembly robot in Section IV-A, which has a power output of about $100 \mathrm{~mW}$ at $6 \mathrm{~V}$, it is clear there is much room for improvement in the motor design.

Assembly of the motor relies extensively on human intervention, so this experiment should be regarded as a proof-ofprinciple that a subset of the CFS (Figure 4) can build working actuators. Topics of current study related to this experiment are 1) incorporating the metal-deposition nozzle into a Reprap machine to print entire coil plates, 2) construction of a motor assembly machine using the modular electro-mechanical components, and 3) increasing power output and eliminating need for permanent magnets.

\section{Fabrication Tool Production}

Figure 13 illustrates a closed manufacturing process for replicating polyurethane cutting tools, which is a subset of the CFS shown in Figure 4. Three types of cutting tool are necessary for the process: a standard endmill, a tapered endmill, and a lathe cutting tool. The process begins with bulk wax material, which is turned and faced into two cylinders using lathe operations. One cylinder receives a conical bore 


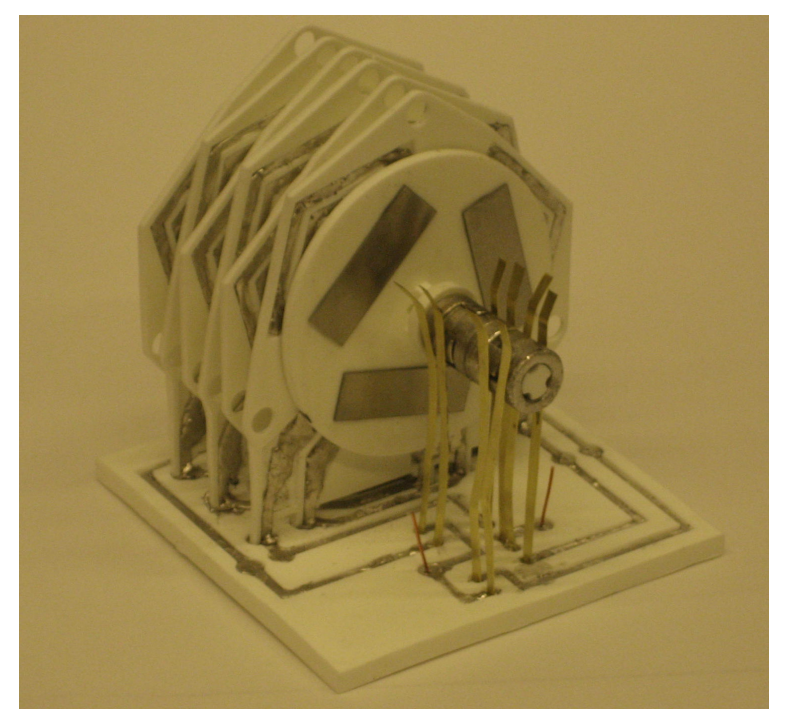

Fig. 11. A DC brush motor fabricated using materials and processes from the cyclic fabrication system. For size reference, the base plate is about $12 \mathrm{~cm}$ square.

and the other a tapered surface. Flutes (cutting surfaces) are cut in the cylinders using milling operations. This results in a wax "master pattern" replica of a standard and tapered endmill. Additional milling operations generate parts for a wax mold container. The master pattern together with the mold container form a pattern from which silicone molds can be cast. The silicone molds contain a negative replica of the wax masters in which polyurethane parts can be cast. The process for replicating lathe cutting tools is simpler - a negative pattern can be machined directly into a wax block using a polyurethane milling tool. This process was verified by using a manually operated milling base (Sherline) to replicate polyurethane cutting tools. An additional subset of the CFS was tested by producing a copper nozzle similar to the one used in the assembly of Figure 9. Results are shown in Figure 12. Materials used in the experiments are: wax (melting point $68 \mathrm{C}$, McMaster P/N 93955K77), polyurethane resin (Smooth-On Smooth-Cast 300), silicone elastomer resin (Smooth-On MoldMax 20), Cerroshield alloy (melting point 95C, McMaster P/N $8921 \mathrm{~K} 23$ ), and aqueous copper sulfate solution.

\section{CONCLUSION}

We have proposed an architecture for a modular robotic system that implements a cyclic fabrication system. The system is intended for use as a general-purpose manufacturing system, with the special feature that it can self-reconfigure and self-replicate. Some of the unique problems encountered in design of self-replicating machines were addressed. All of these problems arise due to the coupling between robot and what it makes - the robot cannot be designed independently of its "product".

Several proof-of-principle experiments supported the feasibility of the cyclic fabrication system. A primary factor in selecting the processes for the CFS is ease of use in

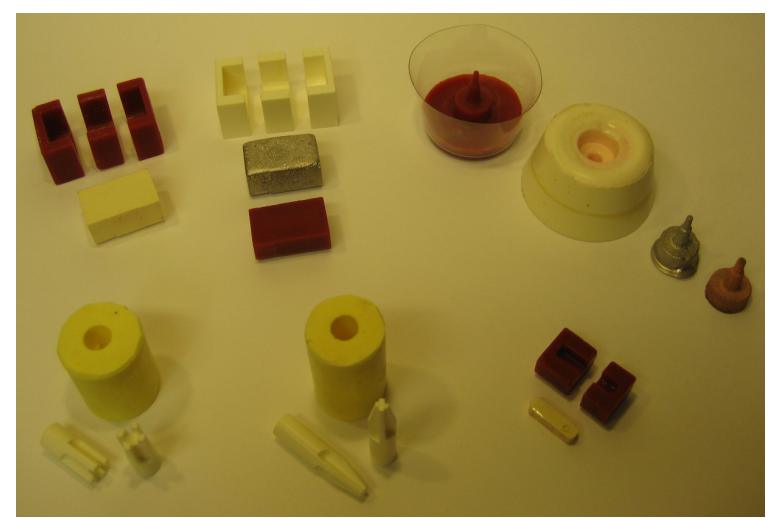

Fig. 12. Clockwise from top left: wax mold, polyurethane part; polyurethane mold, wax and low-melt alloy parts; wax master, polyurethane mold, Cerroshield alloy mandrel, electroformed copper nozzle; wax mold, polyurethane lathe tool; silicone molds and polyurethane end-mills.

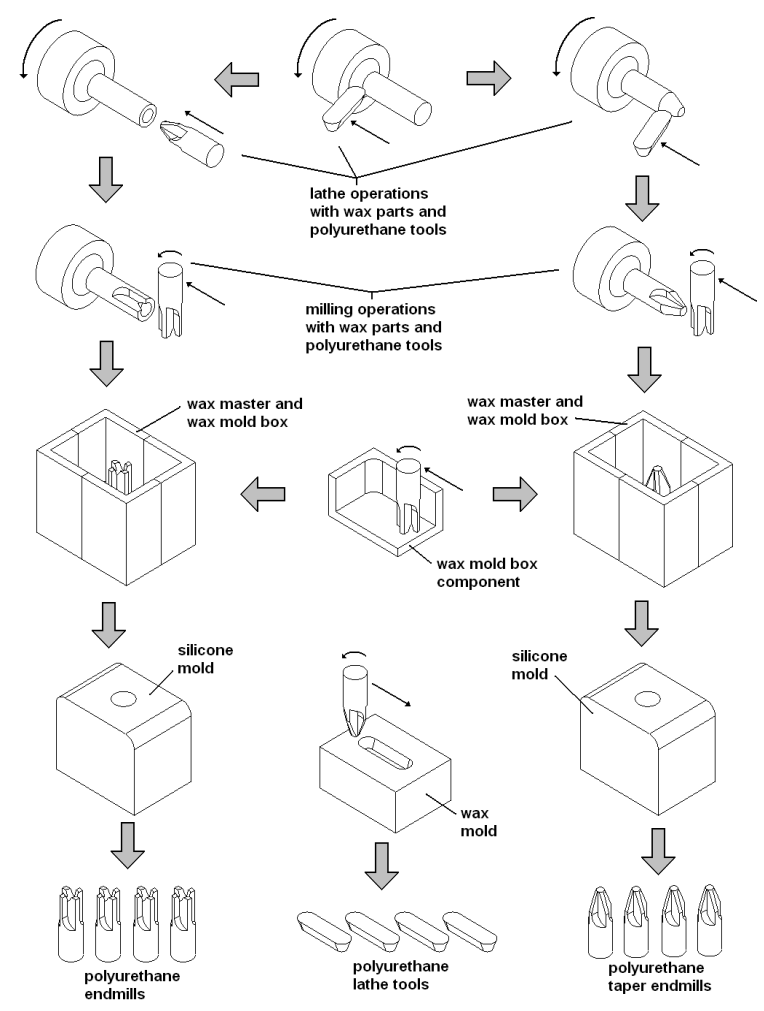

Fig. 13. Process flowchart for replicating cutting tools.

the laboratory. While the exact processes chosen may not be practical for making machines that operate in the "realworld" we argue that the results are useful for several reasons. First, the processes investigated serve as proxies for industrial processes. Design principles uncovered during investigation may be applied to more common methods of manufacturing, for example machineable ceramics and aluminum casting might be used in place of wax and Cerroshield with minor modifications to the basic design of the production cycle. Second, the products generated by the production system as-is may be useful in certain applications, just as the objects built 
by commercial rapid prototyping devices have found use in certain applications, despite a limited selection of materials to work with.

Finally, the most exciting application for a compact automated production system may be for building things small. Silicone elastomer casting and electroforming are routinely used in microfabrication. The physicist Richard Feynman famously suggested using a remotely operated machining system to replicate itself in incrementally smaller and smaller iterations. Admittedly, he called the idea "one weird possibility" and "a very long and very difficult program" [42], but perhaps it may not be so weird or so difficult if approached in the right way.

Topics for future research include: quantifying assembly tolerance functions, incorporating electrical conductors into the modular components, designing new robot assemblies with increased workspace, automating the motor fabrication process, and designing new actuators with improved power output.

\section{ACKNOWLEDGMENT}

The RepRap and Fab@home projects have done a great service by publishing design details and building instructions for their machines in a free and open manner. We thank the large number of volunteers who contribute to these projects.

\section{REFERENCES}

[1] A. Cho, "Making machines that make others of their kind," Science, vol. 318, no. 5853, pp. 1084-1085, 2007.

[2] J. von Neumann and A. W. Burks, Theory of Self-Reproducing Automata. University of Illinois Press, 1966.

[3] "Advnaced automation for space missions," in Proceedings of the 1980 NASA/ASEE Summer Study, R. A. Freitas, Jr., Ed., 1980. [Online]. Available: http://www.islandone.org/MMSG/aasm/

[4] G. S. Chirikjian, Y. Zhou, and J. Suthakorn, "Self-replicating robots for lunar development," IEEE/ASME Transactions on Mechatronics, vol. 7 , no. 4, pp. 462-472, 2002.

[5] T. Bass, "Robot, build thyself," Discover, pp. 64-72, Oct. 1995. [Online]. Available: http://discovermagazine.com/1995/oct/robotbuildthysel569

[6] Reprap main web page. [Online]. Available: http://reprap.org

[7] Fab@home main web page. [Online]. Available: http://fabathome.org

[8] H. Lipson, "Homemade: The future of functional rapid prototyping," IEEE Spectrum, pp. 24-31, May 2005.

[9] R. A. Freitas, Jr. and R. C. Merkle, Kinematic SelfReplicating Machines. Landes Bioscience, 2004. [Online]. Available: http://www.molecularassembler.com/KSRM.htm

[10] M. Sipper, "Fifty years of research on self-replication: An overview," Artificial Life, vol. 4, no. 3, pp. 237-257, 1998.

[11] M. H. Yim, Y. Zhang, and D. G. Duff, "Modular robots," IEEE Spectrum, pp. 30-34, Feb. 2002.

[12] H. Kurokawa, K. Tomita, A. Kamimura, S. Kokaji, T. Hasuo, and S. Murata, "Distributed self-reconfiguration of M-TRAN III modular robotic system," International Journal of Robotics Research, vol. 27, pp. 373-386, 2008.

[13] Z. Butler, K. Kotay, D. Rus, and K. Tomita, "Generic decentralized locomotion control for lattice-based self-reconfigurable robots," International Journal of Robotics Research, vol. 23, no. 9, pp. 919-937, 2004.

[14] S. Griffith, D. Goldwater, and J. M. Jacobson, "Self-replication from random parts," Nature, vol. 437, p. 636, Sep. 2005.

[15] E. Klavins, "Programmable self-assembly," Control Systems Magazine, vol. 24, no. 4, pp. 43-56, Aug. 2007.

[16] K. Tsui, A. A. Geisberger, M. Ellis, and G. D. Skidmore, "Micromachined end-effector and techniques for directed MEMS assembly," Journal of Micromechanics and Microengineering, vol. 14, no. 4, p. 542,2004
[17] K. Lee, M. Moses, and G. S. Chirikjian, "Robotic self-replication in structured environments: Physical demonstrations and complexity measures," International Journal of Robotics Research, vol. 27, pp. 387401,2008

[18] J. Suthakorn, A. B. Cushing, and G. S. Chirikjian, "An autonomous self-replicating robotic system," in Proceedings of 2003 IEEE/ASME International Conference on Advanced Intelligent Mechatronics, 2003.

[19] V. Zykov, E. Mytilinaios, B. Adams, and H. Lipson, "Self-reproducing machines," Nature, vol. 435, no. 7038, pp. 163-164, 2005.

[20] K. J. De Laurentis, C. Mavroidis, and F. F. Kong, "Rapid robot reproduction," IEEE Robotics \& Automation Magazine, vol. 11, no. 2, pp. 86-92, Jun. 2004.

[21] J. G. Cham, S. A. Bailey, J. E. Clark, R. J. Full, and M. R. Cutkosky, "Fast and robust: Hexapedal robots via shape deposition manufacturing," International Journal of Robotics Research, vol. 21, no. 10, Oct. 2002

[22] D. H. Gracias, J. Tien, T. L. Breen, C. Hsu, and G. M. Whitesides, "Forming electrical networks in three dimensions by self-assembly," Science, vol. 289, no. 5482, pp. 1170-1172, Aug. 2000.

[23] U. Pesavento, "An implementation of von neumann's self-reproducing machine," pp. 337-354, 1995.

[24] W. M. Stevens, "Simulating self-replicating machines," Journal of Intelligent and Robotic Systems, vol. 49, no. 2, pp. 135-150, Jun. 2007.

[25] R. Ewaschuk and P. D. Turney, "Self-replication and self-assembly for manufacturing," Artificial Life, vol. 12, no. 3, pp. 411-433, 2006.

[26] A. Menezes and P. Kabamba, "A combined seed-identification and generation analysis algorithm for self-reproducing systems," in Proceedings of the 2007 American Control Conference, Jul. 2007, pp. 2582-2587.

[27] P. D. Owens and A. G. Ulsoy, "Self-reproducing machines: preventing degeneracy," in Proceedings of ASME International Mechanical Engineering Congress and Exposition, Nov. 2006.

[28] W. R. Buckley, "Computational ontogeny," Biological Theory, vol. 3, no. 1, pp. 3-6, 2008.

[29] M. Moses, "Physical prototype of a self-replicating universal constructor," Master's thesis, University of New Mexico, 2001. [Online]. Available: http://home.earthlink.net/ mmoses152

[30] G. S. Chirikjian, Stochastic Models, Information Theory, and Lie Groups. Birkhäuser, 2009.

[31] A. C. Sanderson, "Parts entropy methods for robotic assembly system design," in Proceedings of the IEEE International Conference on Robotics and Automation, 1984, pp. 600-608.

[32] S. Murata, H. Kurokawa, and S. Kokaji, "Self-assembling machine," in Proceedings of the IEEE International Conference on Robotics and Automation, 1994, pp. 441-448.

[33] (2006, Dec.) Mindstorms autofabrik. [Online]. Available: http://www.youtube.com/watch? $=\mathrm{GQ} 3 \mathrm{AcPEPbH} 0$

[34] D. Esterman, M. Sullivan, J. Bergendahl, and C. G. Cassandras, "Computer-controlled lego factory," University of Massachusetts/Amhherst, Tech. Rep., Jun. 1995. [Online]. Available: http://vita.bu.edu/cgc/newlego/index.html

[35] B. Kirby, B. Aksak, S. C. Goldstein, J. F. Hoburg, T. C. Mowry, and P. Pillai, "A modular robotic system using magnetic force effectors," in Proceedings of the IEEE International Conference on Intelligent Robots and Systems, Oct. 2007, pp. 2787-2793.

[36] K. S. Lackner and C. H. Wendt, "Exponential growth of large selfreproducing machine systems," Mathematical and Computer Modelling, vol. 21 , no. 10 , pp. $55-81,1995$.

[37] C. J. Phoenix. (1998, Mar.) Partial design for macro-scale machining self-replicator. [Online]. Available: http://groups.google.com/group/sci.nanotech/msg/96a67c84809c9a5d

[38] E. Malone, K. Rasa, D. L. Cohen, T. Isaacson, H. Lashley, and H. Lipson, "Freeform fabrication of $3 \mathrm{~d}$ zinc-air batteries and functional electromechanical assemblies," Rapid Prototyping Journal, vol. 10, no. 1, pp. 58-69, 2004.

[39] E. Sells and A. Bowyer, "Rapid prototyped electronic circuits," University of Bath, Tech. Rep., Nov. 2004. [Online]. Available: http://staff.bath.ac.uk/ensab/replicator/Downloads/report-01-04.doc

[40] R. Jones. (2009, Apr.) Reprap solder extruder. [Online]. Available: http://www.youtube.com/watch?v=Wnu4lmOnX00

[41] A. E. Fitzgerald, C. Kingsley, and S. D. Umans, Electric Machinery, 6th ed. McGraw-Hill, 2003, pp. 30-42.

[42] R. P. Feynman. (1959) There's plenty of room at the bottom. [Online]. Available: http://www.zyvex.com/nanotech/feynman.html 